\title{
A Synthetic Actor Model for Long-Term Computer Games
}

Danielle R. Silva, Clauirton A. Siebra, Jeferson L. Valadares, Alessandro L. Almeida, Alejandro C. Frery, Jorge da Rocha Falcão ${ }^{1} \&$ Geber L. Ramalho

\author{
Centro de Informática e Departamento de Psicologia ${ }^{1}$ \\ Universidade Federal de Pernambuco \\ Caixa Postal 7851 - CEP 50732-970 - Recife (PE) - Brasil \\ $\{d r d s, c a s, j l f v, a l l$, frery, glr\}@cin.ufpe.br \\ and jtrf@npd.ufpe.br
}

\begin{abstract}
Virtual Reality and Artificial Intelligence provide suitable techniques to improve computer games quality. While the former offers mechanisms to model environment and characters physical features, the latter provides models and tools for building characters, namely Synthetic Actors or Believable Agents, which can exhibit intelligent social behavior and express personality and emotions. The current architecture proposals for Synthetic Actors do not fully meet the requirements for long-term games development. In long-term games, such as strategy and adventure ones, it is necessary to guarantee both personality stability and reactive emotional responses, which may be contradictory. In this work, we propose a new Synthetic Actor model that tightly connects emotions and social attitudes to personality, providing a longterm coherent behavior. This model has been applied to two games presented here as case studies.
\end{abstract}

Keyword: intelligent agents, synthetic actors, computer games, and personality.

\section{Introduction}

The design of computer games must consider the following issues: the interface display, the game playing and the characters' behavior [2]. Virtual Reality can improve both interactivity and interface display aspects, augmenting games realism [3]. In fact, this technique provides mechanisms for the user to immerge, navigate and interact with the surrounding game environment. In order to further increase realism, intelligent agents paradigm and techniques have been used to model the game's characters, giving them the capability of expressing emotions, personality and goaldriven behavior. These characters, also known as Synthetic actors (SA) or Believable Agents, act autonomously, exhibiting behavior which, from a human player's standpoint, is indistinguishable from the behavior of characters controlled by other human players. 
Several SA models have been proposed ([4], [5], [6] and [7]). However, they do not fully meet the requirements of long-term games whose duration usually exceeds a dozen hours. In long-term games, such as strategy and adventure ones, it is necessary to guarantee both personality stability and reactive emotional responses, issues that can lead to contradictory choices.

In this paper, we analyze some current SA models and propose a new one, which provides a more structured use of personality in decision-making for long-term games. The next Section discusses the role of personality, emotion and social behavior in modeling SAs. Section 3 presents a critical review on state of the art. Section 4 introduces our SA model, discussing the underlying assumptions, how it works, and how its components are represented. Section 5 gives an overview of the implementation of two games which are both support and a test-bed of our SA model. Conclusions and future work are presented in the last Section.

\section{Role of Personality, Emotion and Social Behavior in Synthetic Actors}

It is well known that the believability of games' characters can strongly influence the level of computer games realism [8]. In this context, character believability refers to SA consistent behavior when interacting with human players or other SAs. Unfortunately, it is hard to define and implement character believability.

To address this issue, game developers have observed and learned from other media professionals [9]. In fact, computer games bear similarities with traditional media such as TV, films or animated cartoons. In both cases, there is a background story, several characters that interact with each other, and a main goal, which is to entertain the user. The main difference between traditional entertainment media and computer games is that here the user can interact, causing unpredictable changes in the plot. Consequently, the games' characters believability can only be achieved by making them capable of autonomously managing and expressing personality, emotions and social behavior [10]. Human professionals guarantee the character believability in traditional media during film or cartoon production. As discussed in the following Section, the autonomy of these Synthetic Actors has been implemented by modeling them as intelligent agents.

Personality is generally considered a crucial feature to make SA more lifelike, since it is expressed in terms of behavior patterns that distinguish an individual from others [11]. Personality has a strong influence on emotion expression, social behavior and decision-making, because it evokes expectations that keep the user involved in the story ([12] and [7]). Personality must be strongly and reliably represented, and it may be expressed in several ways, e.g. text, facial expression, graphics and so on. The importance of personality has been well understood by cartoonists and filmmakers such as Walt Disney (C) and Warner Bros. (C), who obtained impressive results with some of their characters (e.g., Goofy and Bugs Bunny, respectively).

In order to create illusion of life, SAs must also exhibit proper emotions according to the facts of the story plot. Expressing emotions, such as ache, joy or rage, increases 
the possibility of agents' reactions and personality traits being perceived by the users [13].

Finally, SAs believability can be also improved by an adequate social behavior, i.e., a coherent relationship among the game characters. In fact, an SA must exhibit specific attitudes towards other SAs and environment objects, so that these attitudes guide the interactions (negotiation, dispute, coordination and cooperation) [13].

\section{State of the Art}

The current approaches to synthetic actors modeling can be compared according to different criteria, such as decision-making process (reaction or planning), output (text or graphics), knowledge representation (rules, frames, neural nets, etc.), and believability degree and implementation complexity. In this Section, we briefly present the current SA architectures, focusing on some believability issues.

\subsection{Current SA architectures: shared features}

The existing models for SAs are, in majority, based on the classic agent architecture. In this architecture, a sensorial system continuously acquires environment information; a inference mechanism selects actions considering the sensorial data, and the agent's goals and internal state; and an ensemble of effectors executes actions [14]. The internal state, which can be a representation of both the external environment and the agent's mood, is updated at each inference cycle. The main components explicitly represented in almost all SA models are the folowing:

$\square$ Goals that denote desirable states of the environment (e.g., all cities of the world were conquered, all enigmas were solved, etc.).

$\square$ Mood that designates the emotional and physical state of the SA. In most of the current systems, mood affects the goals and/or action selection. For instance, the feeling of fear can make an SA give up its main goal and run away.

$\square$ Attitudes that are interpersonal relations between SAs or an SA and the environment. For instance, a synthetic actor representing a cat will act differently depending on what it has seen: a dog or a mouse.

$\square$ Personality that is modeled as personal behavior patterns. For instance, a lazy agent will escape its obligations. In the proposed SA architectures, personality is usually modeled as an array of traits to which numerical values are assigned. For example, friendly/unfriendly.

Despite these shared features, there are several differences among the current SA models. Most of these differences concern how personality influences (or is influenced by) other variables (mood, attitudes and goals); how all these variables are influenced by the environment events; and how all these variables influence the decision-making (action selection) process.

Following, we briefly review three SA-related projects, which are the most relevant to the games we are currently developing (Cf. Section 5.1). For each project, we sketch the mutual influence among personality traits, mood, attitudes and goals. 


\subsection{The OZ Project}

The OZ project [4] aims at building synthetic actors that exhibit goal-driven behavior in interactive environments. The model of emotions is based on the work of Orthony, Clore and Collins [15]. The authors propose an architecture called TOK, where emotion is the main channel for expressing an actor's behavior pattern. The values of the personality traits (such as curiosity, happiness, and aggressiveness) evolve by comparing the external pairs events/goals, actions/standards and objects/attitudes.

Fig. 1 gives a sketch of the reasoning flow involving the perceptual data, personality, mood, attitudes, goals and actions. The perceptual data may be used to modify the mood intensity, which will in turn induce changes on the personality traits and attitudes. Goal selection is performed according to the current values of the personality traits, mood and attitudes. Finally, the selected goal coupled with the personality traits and attitudes determine the action the SA will perform. As it might be expected, the TOK model also considers reactions through arc-reflex liaisons between perception and action.

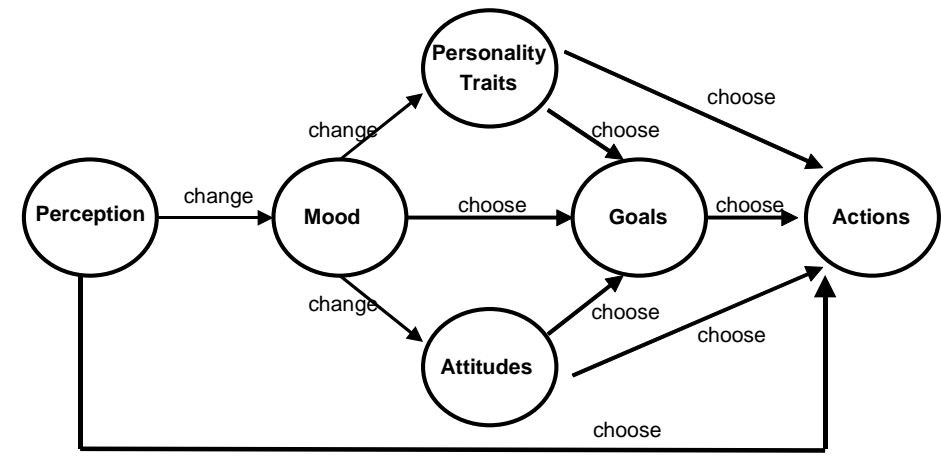

Fig. 1. Tok schematic model.

\subsection{The Virtual Theater Project}

The Virtual Theater Project [7] implements agents that behave like intelligent actors portraying fictitious characters, improvising their behavior rather than having detailed plans. In this project, the personality model is based on trait [16] and social learning [17] theories. The components of the social-psychological model are mood, attitudes and personality traits (Cf. Fig. 2). The values of personality traits, which play a central role on the reasoning process, are influenced by emotions and attitudes, as well as by perceptual data. Personality traits and perceptual data are taken into account in the selection of the actions to be performed at each inference cycle. 


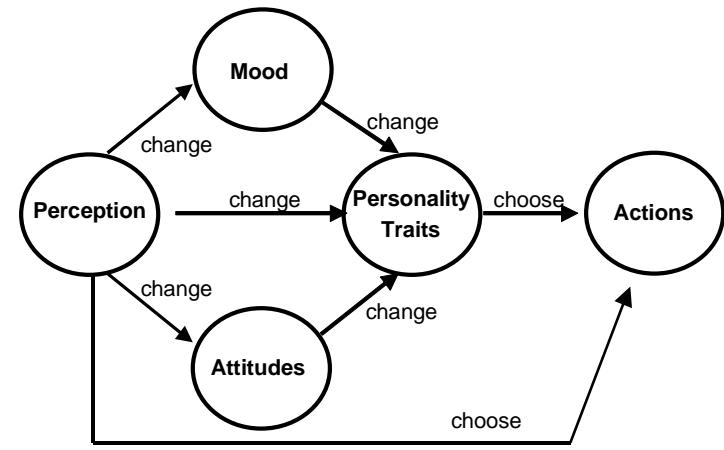

Fig. 2. Virtual Theater schematic model

\subsection{The GULL Project}

The SA model of the GULL project [6] was motivated by the limitations of the previous models, where personality was either modeled as a straight mapping from moods to actions or built on the basis of a simple combination of personality traits. This project is based on a theory claiming that personality is a coherent pattern of behaviors and interactions with the environment [18]. To implement this theory, each personality assigns priorities to a given set of goals (Cf. Fig. 3). For instance, an altruist actor aims to give others what they need and receive positive evaluation of its behavior. The plans (sequence of actions) are chosen taking into account the goal with highest priority and the perceptual data.

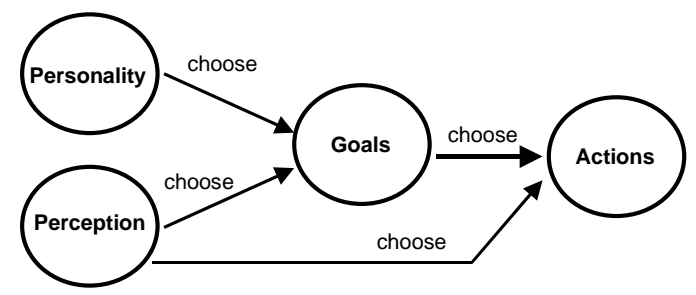

Fig. 3. GULL schematic model

\subsection{Criticism}

An important characteristic of the TOK and Virtual Theater models is that personality traits can be modified abruptly according to mood and attitude changes. These models were tested on applications whose interaction time is considerably short (typically less than half an hour). In these cases, fast personality changes may not be crucial for game credibility. However, in long-term games, such as strategy and adventure ones, 
characters must exhibit consistent and persistent personality, since a stable personality provides a better identification of the character's behavior patterns [9]. This is the case of cartoon characters whose caricature-based, never-changing personality helps to improve the credibility level.

The psychology community shares this interpretation of personality as a structural long-term characteristic. In fact, some psychologists view personality as a complex human factor that influences the emotion, perception of the environment and cognitive processes [11]. Personality may slightly change, but this process is a longterm one, not directly connected to transitory changes on the emotional state.

The GULL project model conceives personality as a time-invariant element whose definition does not depend on other components, such as emotion or attitudes. This model guarantees behavior coherence, which improves believability in long-term applications. However, by excluding mood and attitudes, this model neglects two important factors in creating the illusion of life.

The challenge in long-term games is to ensure personality stability as well as prompt reactive emotional responses and social behavior, which may be contradictory sometimes. The problem of implementing both stable behavior and reactive responses is similar to the classical discussion on (long-term) planning versus pure reactive actions [14]. It is necessary to keep track of the long-term goals. However, in unexpected situations, reactive actions are needed to guarantee a robust behavior. In other words, sometimes an SA must be able to temporarily exhibit a mood, which is incompatible with its personality.

\section{Our Model}

In this Section, we introduce a new SA model that tries to combine a steadily consistent personality with short-term emotions and attitudes in response to transient facts.

\subsection{Overview}

Fig. 4 depicts the relationship among the main concepts of our model, where personality plays a central role.

As in the Virtual Theater, we represent personality as an ensemble of traits whose values are defined by the user according to the particular personality instance he or she wants to create. However, our personality model is based on the Big Five Theory [19], according to which the five main classes of personality traits are: agreeableness, friendliness, consciousness, emotional stability, and openness (Cf. Section 4.2.2).

Personality influences mood and attitude values by defining their initial and default values. For instance, let us suppose that the user assigns a friendly personality to a given actor. This assignment automatically sets a high value for the happiness feature of this actor. The recurrent arrows in mood and attitudes of Fig. 4 indicate a timedependent regulation mechanism which forces their values to get closer to the default ones. For instance, if a tragic event induces temporary sadness on the friendly actor, then, as time goes by, its happiness will go back to the high level. Furthermore, this 
actor will not be angry with another actor for a long time, no matter what the latter has done.

Personality also influences mood and attitudes by amplifying or attenuating the impact of the environment perceptual data. For instance, if a coward synthetic soldier meets a strong enemy, its fear level increases more than if it was a courageous one.

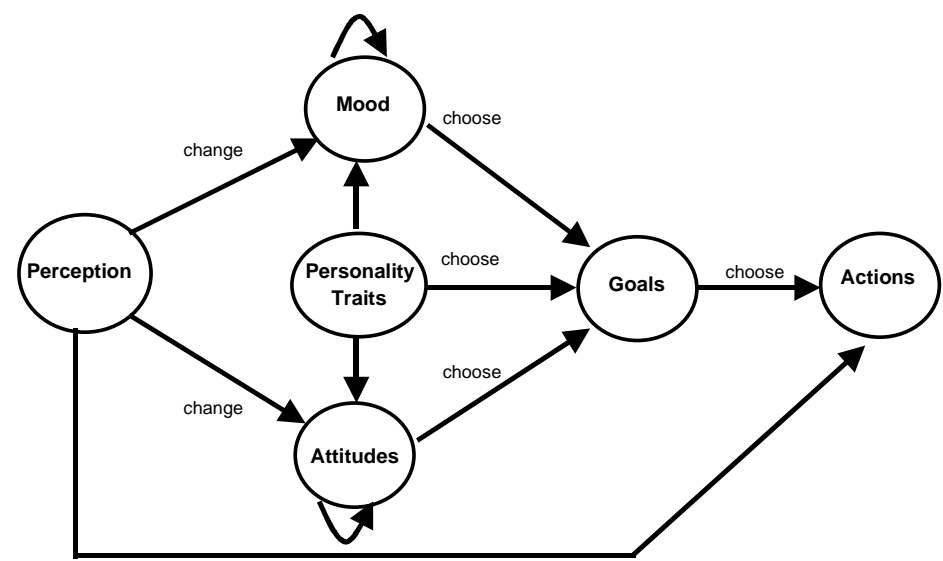

Fig. 4. Our personality model

In our model, the first reasoning step is responsible for updating the SA mood and attitudes according to sensory perception and personality trait values. The second step concerns the selection of the goals to be achieved as a function of mood, attitudes and personality. Finally, taking into account the perceptual data and the goals, the actions or plans are selected.

These steps are carried out by production rules stored in three knowledge bases [14]. In the first knowledge base, rule preconditions take into account perceptual information and personality traits, whereas rule conclusions determine how mood and attitudes vary. An example of such rules is: "if the agent is sensitive (personality trait) and it witnesses its friend killing another agent (sensing), then it will become very sad (mood), and the friendship (attitude) toward its friend will be diminished".

The second knowledge base contains rules whose preconditions concern mood, attitudes, and personality traits to determine the goal. Some examples of these rules are: "if the agent is fatigued (physical mood) or bored (emotion) but it is perseverant (personality trait), then it will keep its current goals" and "if the agent does not trust the other agents, but it is optimistic (personality trait), then it will be ready to negotiate with them".

The rules of the third knowledge base simply instantiate the goal into concrete actions according to the perceptual data. For instance, "If an agent whose goal is to negotiate with agents of a given ethnic group meets another agent of this group, then it will negotiate with the latter". 


\subsection{Components representation}

As we presented previously, the three main psychological components in the agents' cognitive process are mood, attitudes and personality traits. We represent these components similarly to the Virtual Theatre. The main difference is the fact that we model them according to the Object-Oriented paradigm in order to improve reusability and modularity.

\subsubsection{Mood}

Mood is represented by the object SAMood containing objects named SAMoodFeature, whose attributes are name and intensity, defined along a [-10, 10] axis. Opposite mood features are represented by the same object, according to different values of intensity. For instance, "joy" is designated by an instance of SAMoodFeature, whose name is "joy", and whose intensity is a value close to 10 , whereas the same object represents sadness with an intensity value close to -10 . Fig. 5 shows the attributes and methods of these two objects, using UML notation [1]. So far, the features considered in our projects are joy/distress, fatigued/excited, calm/nervous, fear/confidence, and satisfaction/disappointment.

\begin{tabular}{|c|c|c|c|c|}
\hline $\begin{array}{l}\text { ksa>> } \\
\text { SAMoodlnterface }\end{array}$ & \multirow{3}{*}{ implements } & $\begin{array}{l}<<\text { sa }>> \\
\text { SAMood }\end{array}$ & \multirow{2}{*}{ is compose of } & $\begin{array}{l}<<\text { sa }> \\
\text { SAMoodFeature }\end{array}$ \\
\hline & & \multirow[t]{2}{*}{ pMood } & & $\begin{array}{l}\text { pFeatureName } \\
\text { plntensity }\end{array}$ \\
\hline $\begin{array}{l}\operatorname{addMood}(\ldots) \\
\text { removeMood(...) }\end{array}$ & & & & $\begin{array}{l}\text { getMoodName() } \\
\text { getMoodIntensity() }\end{array}$ \\
\hline
\end{tabular}

Fig. 5. Mood representation

\subsubsection{Personality}

Similarly to mood, personality is represented by an integration object (SAPersonality), which contains objects (SAPTrait) designating its traits (Cf. Fig. 6). Each trait is represented by a name and an intensity, which ranges from -10 to 10 according to the desired quality (e.g., cowardice/courage and aggressiveness/rationality). Although the objects of the class SAPTrait contain methods for setting intensity values, these values are invariable along the game, as discussed previously. 


\begin{tabular}{|c|c|c|c|c|}
\hline $\begin{array}{l}<<\text { sa }>> \\
\text { SAPTraitlnterface }\end{array}$ & & $\begin{array}{l}<<\text { sa }> \\
\text { SAPersonality }\end{array}$ & & $\begin{array}{l}\text { <<a }>> \\
\text { SAPTrait }\end{array}$ \\
\hline & implements & & is compose of & $\begin{array}{l}\text { pTraitName } \\
\text { plntensity }\end{array}$ \\
\hline $\begin{array}{l}\text { addPTrait }(\ldots) \\
\text { removePTrait (...) }\end{array}$ & & & & $\begin{array}{l}\text { getTraitName() } \\
\text { getTraitIntensity() }\end{array}$ \\
\hline
\end{tabular}

Fig. 6. Personality traits representation

Based on the Big Five Theory [19], [19], we have taken into account the following personality traits classes:

$\square$ Friendliness that indicates whether an individual is naturally nice or hostile towards the others.

$\square$ Openness that concerns agent's interests. High openness refers to characters with lots of shallow interests, whereas low openness refers to an SA with few and deep interests;

$\square$ Emotional stability that defines the sensibility level of SAs with respect to their perception. Resilient SAs are only bothered by strong stimuli, whereas reactive SAs are bothered by a greater variety of stimuli;

$\square$ Agreeableness that concern characters self-confidence. High agreeableness applies to SAs that follow social conventions, whereas low agreeableness describes an SA that, in the extreme, only follows its own rules;

$\square$ Consciousness refers to the number of goals a character is focused on. Highly conscientious SAs focus on few goals and exhibit the self-discipline associated with such focus. Low conscientiousness refers to an SA that pursues a larger number of goals, exhibiting spontaneity.

\subsubsection{Attitudes}

Attitudes are represented by the object SAAttitudes, containing an ensemble of individual attitudes (SAAttitude), which are defined by a name, an intensity and a target (other agents or objects) to which the attitude refers. Fig. 7 shows these two objects. So far some attitudes considered in our projects are like/dislike, trust/distrust, admire/reproach. 


\begin{tabular}{|c|c|c|c|c|}
\hline \multirow[t]{2}{*}{$\begin{array}{l}\text { K<sa>> } \\
\text { SAAtInterface }\end{array}$} & \multirow{3}{*}{ implements } & $\begin{array}{l}\ll \text { sa }>> \\
\text { SAAttitudes }\end{array}$ & \multirow{2}{*}{ is compose of } & $\begin{array}{l}<<\text { sa }>> \\
\text { SAAttitude }\end{array}$ \\
\hline & & pAttitudes & & $\begin{array}{l}\text { pAttitude } \\
\text { pObject }\end{array}$ \\
\hline $\begin{array}{l}\text { addAttitude (...) } \\
\text { removeAttitude (...) }\end{array}$ & & & & $\begin{array}{l}\text { getAttitude() } \\
\text { getObject() }\end{array}$ \\
\hline
\end{tabular}

Fig. 7. Attitudes representation

\section{Implementation \& Results}

Motivated by the possibilities of bringing together Artificial Intelligence and Virtual Reality in the context of computer games, we are currently developing two computer games: Enigmas in the Campus [21] and Guararapes [22], where the characters are represented by our synthetic actor model.

\subsection{Game projects}

Enigmas in the Campus (Fig. 8a) is an adventure game set in the virtual campus of our University. In order to improve motivation and engagement for exploring this virtual environment, the game offers enigmas to be solved by the players. There are some fictitious characters in the game. For instance, the guardians are synthetic actors that represent wise creatures having knowledge about the university courses. Their personality traits define how, when and which players they will help during the game. The other type of agents, the novices, is controlled by the user and may interact with the guardians and other novices.

Guararapes (Fig. 8b) is a strategy game aiming to entertain the user while providing information about the historical XVII century Brazilian battle of Guararapes [23]. In Guararapes, the player controls one of the four ethnic groups involved in the dispute, namely: Portuguese, Dutch, Brazilian Indians and Brazilian Africans (African slaves and their descendants). The player will develop strategies that help his/her ethnic group to achieve its goals. The other ethnic groups may be controlled either by humans or by the computer. Agents' personalities, mood and attitudes will influence interactions between them, such as negotiation and dispute. These interactions add social elements, which are essential in strategy games.

The prototypes of Guararapes and Enigmas in the Campus have been implemented in Java ${ }^{\mathrm{tm}}$ (JDK 1.2 e Java3D API) and VRML (Virtual Reality Modeling Language) [25]. The environment was modeled using VRML, and the graphical representations and animations of the characters are being designed in Java3D, a Java graphic API [26] that can improve the efficiency of the system when compared to VRML. The agent reasoning process is implemented through the use of Jeops ([27] and [28]), a first order forward-chaining inference engine that can filter and manipulate Java objects. Java is a suitable language for developing such applications, since, due to its 
object-oriented characteristics, it supports a great number of specialized APIs, including facilities for graphics, networking, reasoning, and so on.
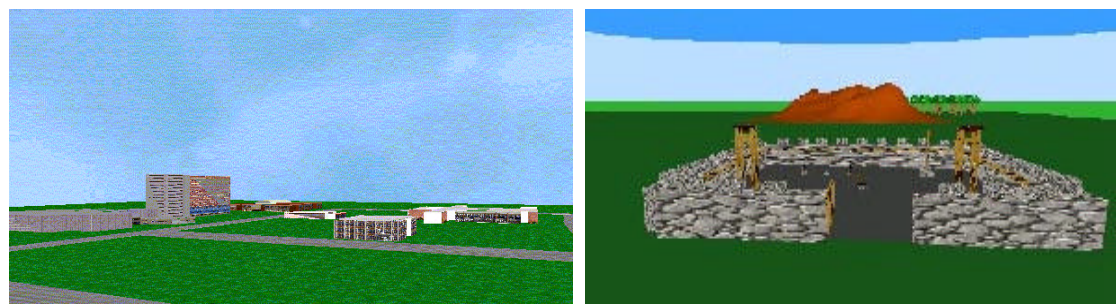

Fig. 8. Scenarios of (a) Enigmas in the Campus and (b) Guararapes, respectively

\subsection{Preliminary results}

The prototypes of our two games are operational. We are performing some evaluations whose goal is to verify to what extent the SA behaviors are compatible with their personality. In other words, the initial experiments try to evaluate whether the spectators can recognize a character personality based only on its behavior.

In Guararapes, the available personality traits are coward/courageous, lazy/dynamic and unfaithful/faithful. In battle situations, users can easily recognize the coward/courageous trait. In these cases, coward characters only fight when facing weak enemies. Otherwise they avoid confrontation at all costs. The lazy/dynamic trait can also be easily noticed by observing how the character moves. Lazy characters walk slowly and prefer plain land instead of forests or hills. The unfaithful/faithful trait should be identified in the negotiation's interactions. Characters that tend to be treacherous are easily seduced to accept proposals, even if this would disappoint their ethnic group. So far, users have not promptly recognized this trait.

In Enigmas in the Campus, the personality is expressed through animations and textual descriptions. For instance, the guardian's colors change when they are happy or sad. Guardians move quickly when they are worried and become transparent when they are weak. Users can easily notice these simple changes. However complex situations and scenarios have not been tested yet.

Our preliminary experiments suggest also that the number of interactions play an important role in the perception of a character's personality. In fact, users may misunderstand some actions when not enough interactions are allowed. For instance, when observing a character running away from a battlefield, the user may think that it is coward. However, this character could simply be moving faster in order to reach a given goal place as soon as possible. The more numerous are the interactions, the more accurate is the user recognition of actors' personalities. This constraint is not a problem to users in strategy and adventure games, since they last several hours. 


\section{Conclusions and Future Plans}

Intelligent agents metaphor and techniques are a promising approach for modeling computer games characters (synthetic actors). However, in order to provide realism and user commitment in long-term games, it is necessary to guarantee some behavioral properties of these actors. In this paper, we proposed an original model for synthetic actors, whose goal is to provide both long-term personality cohesion and short-term emotional reactions. The proposed model is being empirically validated in two games.

Our envisioned future work is centered in a deeper evaluation of our proposed model. This includes the refinement of a methodology to quantitatively assess the opinions of different users and a broad generation of different games scenarios and situations in which the actors' behavior will be evaluated.

\section{References}

[2] Crawford, C. The Art of Game Design. Available in http://www.vancouver.wsu.edu/ fac/peabody/game-book/Coverpage.html. Last consulted in April 2000.

[3] Handcock, D. Viewpoint: Virtual Reality in Search of Middle Ground, IEEE Spectrum 32(1):68. (1995)

[4] Bates, J., Loyall, A. \& Reilly, W. S. An Architecture for Action, Emotion, and Social Behavior. Technical report CMU-CS-92-144, School of Computer Science, Carnegie Mellon University, Pittsburgh. (1992)

[5] Elliott, C. Research problems in the use of a shallow Artificial Intelligence model of personality and emotion. In proceedings of the XII National Conference on Artificial Intelligence (AAAI'94). pp. 9-15, Seattle. (1994)

[6] Rizzo, P., Veloso M., Miceli, M. \& Cesta, A. Personality-Driven Social Behaviors in Believable Agents. In proceedings of the AAAI 1997 Fall Symposium on "Socially Intelligent Agents", Massachusetts. (1997)

[7] Rousseau, D. \& Hayes-Roth, B. Improvisational Synthetic Actors: with Flexible Personalities. Technical report no. KSL 97-10. (1997)

[8] Wilson, I. Artificial Emotion: Simulating Mood and Personality. Gamasutra Online Magazine 3(18), May. (1999)

[9] Laurel, B. Computer and Theater. London: Addison-Wesley Publishing Company. (1999)

[10] Thomas, F. \& Johnston, O. Disney Animation: The Illusion of Life. New York: Abbeville Press. (1981)

[11] Allport, G. W. Concepts of Trait and Personality. Psychological Bulletin, 24, 284-293. (1927)

[12] Reilly, W. S. \& Bates J. Building emotional agents. Technical Report CMU-CS-92-143, School of Computer Science, Carnegie Mellon University, Pittsburgh. (1992)

[13] Reilly, W. S. Believable Social and Emotional Agents. PhD Thesis, School of Computer Science. Carnegie Mellon University. Pitsburgh. (1996)

[14] Russell, S. \& Norvig, P. Artificial Intelligence: a Modern Approach. Englewood Cliffs: Prentice-Hall, Inc. (1995)

[15] Ortony, A., Clore, G. Collins, A. The Cognitive Structure of Emotions. Cambridge University Press. (1988)

[16] Aktinson, R. L., Aktinson, R. C. \& Hilgard, E. R. Introduction to Psychology. San Diego: Harcourt Brace Jovanovich, Inc. (1983) 
[17] Bandura, A. Social Learning Theory. Englewood Cliffs: Prentice-Hall. (1997)

[18] Page, M. Personality: Currently Theory and Research. Lincoln: University Nebraska Press. (1983)

[19] Howard , P. J. \& Howard, J. M. The Big Five Quickstart: An Introduction to the FiveFactor Model of Personality for Human. Available in http://centacs.com/quik-prt.htm. Last consulted in 15 December 2000.

[20] Rumbaugh, Jim; Booch, Grady \& Jacobson, Ivar. The Unified Modeling Language Reference Guide. Addison Wesley Longman. (1999)

[21] Silva, D. Enigma no Campus. Available in http//www.di.ufpe/ drds/enigma/Welcome.html.

[22] Siebra, C. Batalha dos Guararapes. Available in http:// www.di.ufpe.br/ cas.

[23] Bento, C. M. As Batalhas dos Guararapes: descrição e análise militar. Editora Universitária, $2^{\circ}$ Edição, Recife. (1971).

[24] Reeves, B. \& Nass, C. The Media Equation: How people treat computers, Television, and New Media Like People and Places. Cambridge: CSLI Publications. (1996)

[25] Ames, A. L., Nadeau, D. R., and Moreland, J. L. The VRML 2.0 Sourcebook. Wiley, New York. (1996)

[26] Bouvier, D. \& Dankwardt, P. Getting Started with the Java 3DTM API: A Tutorial for Beginners. Sun Microsystems Publishing. (1999)

[27] Figueira Filho, C. \& Ramalho, G. Jeops - the java Embedded Object Production System. In M. Monard e J. Sichman (eds). Advances in Artificial Intelligence. Lecture Notes on Artificial Intelligence Series, vol. 1952, pp 52-61. London: Springer-Verlag. (2000)

[28] Figueira, C. JEOPS User's Manual. Available in http://www.di.ufpe.br/ csff/jeops. Last consulted in April 2000 


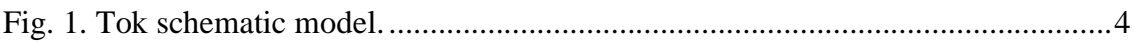

Fig. 2. Virtual Theater schematic model ..........................................................

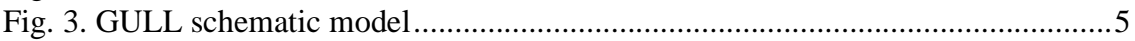

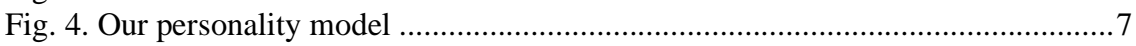

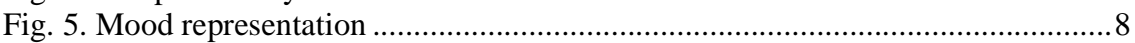

Fig. 6. Personality traits representation ................................................................

Fig. 7. Attitudes representation .................................................................................... 10

Fig. 8. Scenarios of (a) Enigmas in the Campus and (b) Guararapes, respectively.....11 